\title{
COUNTABLY GENERATED IDEALS IN RINGS OF CONTINUOUS FUNCTIONS
}

\author{
LEONARD GILLMAN ${ }^{1}$
}

1. Results. Scattered results about countably ${ }^{2}$ generated ideals in $C(X)$ are established in [2] and [4]: $O^{p}$ is countably generated if and only if $p \in X$ and $p$ has a countable base of neighborhoods; $O^{p}$ is both prime and countably generated if and only if $\boldsymbol{M}^{p}$ is principal, and if and only if $p \in X$ and $p$ is isolated; no lower prime ideal is countably generated. We generalize these as follows: if $\boldsymbol{M}^{p}$ is countably generated, then $p \in X$ and $p$ is isolated (5.4); no free prime ideal is countably generated (4.5); a fixed, nonmaximal prime ideal can be countably generated (6.2), but not if it is a $z$-ideal $(6.1) ;^{3}$ the intersection of a countable chain of lower prime ideals, or of a strictly decreasing sequence of arbitrary prime ideals, cannot be countably generated $(6.3,6.4)$.

Some of the above are corollaries of much more general theorems: any free, countably generated ideal is contained in $2^{\mathfrak{c}}$ hyper-real maximal ideals (4.4); if the fixed ideal of all functions vanishing on a compact set $F$ is countably generated, then $F$ is an open set (5.2). These theorems, in turn, can be formulated more generally. In some of the results, the hypothesis that an ideal is countably generated can be replaced by the weaker hypothesis that its $z$-filter be countably generated.

2. Preliminaries. $C(X)$ denotes the ring of all continuous functions from the completely regular space $X$ into $\mathbf{R}$, the reals. For $f \in C, \boldsymbol{Z}(f)$ denotes the set of zeros of $f$.

Let $I$ be a (proper) ideal in $C$. The family $\boldsymbol{Z}[I]=\{\boldsymbol{Z}(f): f \in I\}$ is a $z$-filter: all finite intersections of members, and all zero-sets containing members, are members; and $\varnothing$ is not a member. Every ideal is contained in at least one maximal ideal [2, Chapter 2].

If $\cap \boldsymbol{Z}[I]$ is nonempty, $I$ and $\boldsymbol{Z}[I]$ are fixed; else, free. The fixed maximal ideals are the sets $\boldsymbol{M}_{p}=\{f: p \in \boldsymbol{Z}(f)\}$, for $p \in X$. More generally, the maximal ideals, free or fixed, are the sets

Presented to the Society, January 29, 1960; received by the editors October 6, 1959.

${ }^{1}$ National Science Foundation fellow. I wish to thank C. W. Kohls for several helpful suggestions.

${ }^{2}$ We use countable to mean finite or denumerably infinite.

${ }^{3}$ Nontrivial $z$-ideals can be countably generated. For example, on a $P$-space, every ideal is a $z$-ideal, and no nonisolated point is a $G_{\delta}$; and there exist $P$-spaces having no isolated points $[1, \S \S 5$ and $7 ; 2,13 \mathrm{P}$ and 14.29]. 


$$
\boldsymbol{M}^{p}=\{f: p \in \operatorname{cl} \boldsymbol{Z}(f)\}
$$

where $\beta X$ is the Stone-Cech compactification of $X$, and "cl" denotes closure in $\beta X$. Related to these are the ideals

$$
O^{p}=\{f: \operatorname{cl} \boldsymbol{Z}(f) \text { is a neighborhood (in } \beta X \text { ) of } p\} \text {. }
$$

If $p \in X, \boldsymbol{M}^{p}=\boldsymbol{M}_{p}$; if $p \in \beta X-X$, then $\boldsymbol{M}^{p}$ and $\boldsymbol{O}^{p}$ are free. $\boldsymbol{M}^{p}$ is the unique maximal ideal containing $O^{p}$. Every prime ideal $\subset M^{p}$ contains $O^{p}[2$, Chapters 4,6 , and 7$]$.

Every $f \in C(X)$ has a continuous extension $f^{*}$ from $\beta X$ into the one-point compactification of $\mathbf{R}[2,6.5]$.

$\mathbf{0}, \mathbf{1}$, etc., denote the corresponding constant functions in $C(X)$.

$\mathbf{N}$ denotes the set (or discrete space) of positive integers $1,2, \ldots$

3. Generators. The ideal $\left(f_{\alpha}, \cdots\right)$ generated by the functions $f_{\alpha}, \cdots$ is the smallest ideal containing every $f_{\alpha}$; it consists of all finite sums $\sum_{k} s_{k} f_{\alpha_{k}}$, where $s_{k} \in C$. The $z$-filter $\left(Z_{\alpha}, \cdots\right)$ generated by the zero-sets $Z_{\alpha}, \cdots$ is the smallest $z$-filter containing every $Z_{\alpha}$; it consists of all zero-sets that contain finite intersections of the $Z_{\alpha}$.

For any ideal $I$, we define

$$
\theta(I)=\left\{p \in \beta X: M^{p} \supset I\right\}, \quad \phi(I)=\left\{p \in X: M_{p} \supset I\right\} .
$$

Evidently, $\phi(I)=\theta(I) \cap X$; and if $I$ is free, then $\phi(I)$ is empty. By (1),

$$
\theta(I)=\bigcap_{f \in I} \operatorname{cl} \boldsymbol{Z}(f), \quad \phi(I)=\bigcap_{f \in I} \boldsymbol{Z}(f) ;
$$

more generally, the intersections may be taken over any set of generators of $I$. Alternatively,

$$
\theta(I)=\bigcap_{\alpha} \mathrm{cl} Z_{\alpha}, \quad \phi(I)=\bigcap_{\alpha} Z_{\alpha},
$$

where $Z_{\alpha}$ ranges over any set of generators of the $z$-filter $\boldsymbol{Z}[I]$.

If $I=\left(f_{\alpha}, \cdots\right)$, then, clearly, $\boldsymbol{Z}[I]=\left(\boldsymbol{Z}\left(f_{\alpha}\right), \cdots\right)$. The converse is false, as we shall see. ${ }^{4}$

When the $z$-filter $\boldsymbol{Z}[I]$ is countably generated, we shall say that the ideal $I$ is countably $z$-generated. Thus any countably generated ideal is countably $z$-generated. When stating the latter condition in the hypothesis of a theorem, we enclose the " $z$-" in parentheses so that the stronger hypothesis will also be in evidence.

The $z$-filter $\boldsymbol{Z}\left[\boldsymbol{M}_{0}\right]$ in $\mathbf{R}$ has a single generator- $\{0\}$-but the ideal $\boldsymbol{M}_{0}$ is not countably generated (5.3). Nor need a free, countably $z$-generated ideal be countably generated (5.5).

4 It holds, however, for the special case $I=O^{p}[2,7 \mathrm{I}]$. 
4. Free ideals. Every residue class field $C / \boldsymbol{M}^{p}$ contains $\mathbf{R}$ (as the set of images of the constant functions). If $C / M^{p}=\mathbf{R}$, then the field, the ideal, and the point $p$ are called real; else, hyper-real. Every point of $X$ is real: a point $p$ of $\beta X$ is hyper-real if and only if there exists $f$ for which $f^{*}(p)=\infty[2$, Chapters 5 and 7$]$.

$c$ denotes the cardinal of the continuum.

4.1. Theorem. A countably (z-)generated ideal contained in a hyperreal maximal ideal is contained in $2^{\mathfrak{c}}$ hyper-real ideals.

Proof. Let $\boldsymbol{Z}[I]=\left(Z_{1}, Z_{2}, \cdots\right) \subset \boldsymbol{Z}\left[\boldsymbol{M}^{p}\right]$, where $p$ is hyper-real. Choose $f \geqq 0$ with $f^{*}(p)=\infty$, and define

$$
F_{n}=\{x \in X: f(x) \geqq n\} \quad(n \in \mathbf{N}) .
$$

Then $F_{n} \in \boldsymbol{Z}\left[\boldsymbol{M}^{p}\right]$, so that all finite intersections of sets $Z_{m}$ and $F_{n}$ are nonempty. Choose a set $D$ of points $x_{n}$ satisfying

$$
x_{n} \in Z_{1} \cap \cdots \cap Z_{n} \cap F_{n}
$$

and $f\left(x_{1}\right)<f\left(x_{2}\right)<\ldots$. Each $Z_{n}$ contains all but finitely many points of $D$, so that cl $D-D \subset \operatorname{cl} Z_{n}$. By (2), cl $D-D \subset \theta(I)$. Also, $f^{*}(q)=\infty$ for each $q \in \operatorname{cl} D-D$, so that every point of $\operatorname{cl} D-D$ is hyper-real.

Evidently, $f$ carries $D$ homeomorphically onto a closed copy of $\mathbf{N}$ in $\mathbf{R}$. By $[2,1.19], D$ is $C^{*}$-embedded in $X$, so that $\mathrm{cl} D-D$ is homeomorphic with $\beta \mathrm{N}-\mathrm{N}[2,6.9(\mathrm{a})]$ and therefore of cardinal $2^{\mathrm{c}}[2,9.2]$.

It follows, by the way, that $\operatorname{cl} D-D$, and hence $\theta(I)$, contains $a$ copy of $\beta \mathrm{N}$ itself $[2,6.10(\mathrm{a})]$.

4.2. Lemma. If a maximal ideal $\boldsymbol{M}^{p}$ contains a countably (z-)generated ideal I such that $p \notin \mathrm{cl} \phi(I)$, then $M^{p}$ is hyper-real.

Proof. Let $\boldsymbol{Z}[I]=\left(Z_{1}, Z_{2}, \cdots\right)$. If $M^{p}$ is real, then by $[2,5.15(\mathrm{a})]$, $\phi(I)=\bigcap_{n} Z_{n} \in \boldsymbol{Z}\left[\boldsymbol{M}^{p}\right]$. By (1), $p \in \mathrm{cl} \phi(I)$, contradicting hypothesis.

4.3. Corollary. If $I$ is countably (z-)generated and $\phi(I)$ is compact, then every free maximal ideal containing $I$ is hyper-real.

The following is a special case of the combination of the theorem with the lemma.

4.4. Theorem. Each free, countably (z-)generated ideal is contained in $2^{\mathfrak{c}}$ hyper-real maximal ideals but in no real maximal ideal.

For, any ideal $I$ is contained in at least one maximal ideal; and if $I$ is free, then $\phi(I)$ is empty.

4.5. Corollary. A free ideal contained in a unique maximal ideal cannot be countably (z-)generated. In particular, no free $\mathrm{O}^{p}$, no free 
prime ideal, and no free maximal ideal are countably (z-)generated. ${ }^{5}$

Regarding 4.4, we remark that if $X$ is realcompact [2, Chapter 8], then any free ideal contained in infinitely many maximal ideals is contained in $2^{\mathrm{c}}$ hyper-real ideals. (Note that $\theta(I)$ is an infinite compact set in $\beta X-X$, and apply $[2,9.11]$.)

5. Maximal ideals. Any compact space is normal. Hence, given two disjoint compact sets in $\beta X$, there exists $s \in C(X)$ such that $s^{*}=0$ on a neighborhood (in $\beta X$ ) of one of the sets, while $s^{*}=1$ on a neighborhood of the other [2, Chapter 3].

5.1. Lemma. If $s^{*}$ vanishes on a neighborhood of $\theta(J)$, then $s \in J$.

Proof. Evidently, $\operatorname{cl} \boldsymbol{Z}(s)$ is a neighborhood of $\theta(J)$. Since $\beta X$ is compact, (2) implies that $\operatorname{cl} \boldsymbol{Z}(s)$ is a neighborhood of $\operatorname{cl} \boldsymbol{Z}(f)$ for some $f \in J$. By $[2,1 \mathrm{D}], s$ is a multiple of $f$.

If $K$ is the ideal of all functions that vanish on a closed set $F$, then $K=\bigcap_{p \in F} M_{p}$, and $\phi(K)=F .^{6}$

5.2. Theorem. Let $F$ be a compact set in $X, K$ the ideal of all functions that vanish on $F$, and $J$ an arbitrary free ideal or all of $C(X)$; if $I=J \cap K$ is countably generated, then $F$ is open.

Proof. Since $J$ is free, the compact set $\theta(J)$ is disjoint from $X$ and hence from $F$. (For $J=C, \theta(J)=\varnothing$.)

Let us verify that $\phi(I)=F$. Evidently, $\phi(I) \supset \phi(K)=F$. On the other hand, given any $p \in X-F$, there exists $s \in C$ such that $s(p)=1$, while $s^{*}$ vanishes on a neighborhood of $F \cup \theta(J)$. Then $s \in K$, and, by the lemma, $s \in J$. Therefore $s \in I$. Since $s \notin M_{p}$, we have $I \nsubseteq M_{p}$, that is, $p \notin \phi(I)$.

Next, choose $t \in C(X)$ such that $t=1$ on a neighborhood $U$ of $F$, while $t^{*}$ vanishes on a neighborhood (in $\beta X$ ) of $\theta(J)$. By the lemma, $t \in J$.

Let $I=\left(f_{1}, f_{2}, \cdots\right)$, where, as we may assume, each $\left|f_{m}\right| \leqq 1[2$, 2A]. Define

$$
h=\sum_{m} 2^{-m}\left|f_{m}\right|^{1 / 2}
$$

Because of the uniform convergence, $h$ is continuous. Now define

$$
g=t h \text {. }
$$

${ }^{5}$ For $O^{p}$, this also follows by combining the first theorem quoted in $\S 1$ with the result stated in footnote 4 .

${ }^{6}$ For realcompact $X$, such ideals are precisely the kernels of the homomorphisms from $C(X)$ into function rings $[2,10 \mathrm{D}]$. 
Since $t=1$ on $U, g$ agrees with $h$ on $U$.

Next, $\boldsymbol{Z}(h)=\bigcap_{m} \boldsymbol{Z}\left(f_{m}\right)=\phi(I)=F$, so that $h \in K$. (Here we need only that $\phi(I) \supset F$.) Since $t \in J$, we have $g \in J \cap K=I$. Hence there exist $n \in \mathrm{N}$ and $s_{1}, \cdots, s_{n} \in C$ such that

$$
g=s_{1} f_{1}+\cdots+s_{n} f_{n} \text {. }
$$

Since $F$ is compact, there exists a neighborhood $V$ of $F$, with $V \subset U$, on which each $\left|s_{k}\right|$ is bounded-say by $r>0$. Then

$$
h(x)=g(x) \leqq r\left(\left|f_{1}(x)\right|+\cdots+\left|f_{n}(x)\right|\right) \quad(x \in V) .
$$

Since each $f_{k}$ vanishes on $F$, there is a neighborhood $W$ of $F$, with $W \subset V$, such that $\left|f_{k}(x)\right|^{1 / 2}<2^{-n} r^{-1}(k \leqq n, x \in W)$. Hence

$$
r\left|f_{k}(x)\right| \leqq 2^{-n}\left|f_{k}(x)\right|^{1 / 2} \quad(k \leqq n, x \in W),
$$

with strict inequality in case $k$ and $x$ satisfy $f_{k}(x) \neq 0$. For such $x$, we would have, by (4),

$$
h(x)<2^{-n}\left(\left|f_{1}(x)\right|^{1 / 2}+\cdots+\left|f_{n}(x)\right|^{1 / 2}\right) .
$$

But this is impossible, in view of the definition of $h$. Consequently, each $f_{k}(k \leqq n)$ must vanish everywhere on $W$. By (3), $g$, and hence $h$, vanishes everywhere on $W$. Thus, $W \subset \boldsymbol{Z}(h)=F$. Since $W$ is a neighborhood of $F$, this implies that $F$ is open.

Example. The ideal in $C(\mathrm{R})$ of all functions that vanish on a given compact set is not countably generated.

5.3. CoROllaRy. If $\boldsymbol{M}_{p_{1}} \cap \cdots \cap \boldsymbol{M}_{p_{n}}$ is countably generated, or if the intersection of this ideal with any free ideal is countably generated, then $p_{1}, \cdots, p_{n}$ are isolated.

5.4. Corollary. If $M^{p}$ is countably generated, then $p \in X$ and $p$ is isolated.

Proof. Corollaries 4.5 and 5.3.

As we know, countably generated cannot be replaced here by countably z-generated.

5.5. ExAmple. A free, countably z-generated ideal that is not countably generated. Let $I$ be the ideal of all functions in $C(\mathbf{R})$ that vanish at all but a finite number of points of $\mathbf{N}$; then $I$ is free. Clearly, the complements in $\mathbf{N}$ of the finite subsets of $\mathbf{N}$ form a countable family of zero-sets in $\mathbf{R}$ that generate $\boldsymbol{Z}[I]$.

We assume that $I=\left(f_{1}, f_{2}, \cdots\right)$ and derive a contradiction. For each $m \in \mathbf{N}$, let $E_{m}$ denote the closed interval $[m, m+1 / 2]$, and let $M_{m}$ denote the maximal ideal in $C\left(E_{m}\right)$ of all functions in $C\left(E_{m}\right)$ that vanish at $m$. Since $M_{m}$ is not countably generated (5.3), there exists a 
function $g_{m} \in C\left(E_{m}\right)$ that belongs to $M_{m}$ but not to the ideal in $C\left(E_{m}\right)$ generated by those functions $f_{k} \mid E_{m}$ that vanish at $m$.

Let $g$ be a function in $C(\mathbf{R})$ that agrees with each $g_{m}$ on $E_{m}$. Then $g$ vanishes everywhere on $\mathbf{N}$, so that $g \in I$. Hence there exist $n \in \mathbb{N}$ and $s_{1}, \cdots, s_{n} \in C(\mathbf{R})$ such that $g=s_{1} f_{1}+\cdots+s_{n} f_{n}$. Since each $f_{k}$ vanishes at all but finitely many points of $\mathbf{N}$, there exists $m \in \mathbf{N}$ at which $f_{1}, \cdots, f_{n}$ vanish simultaneously. But this contradicts $g \mid E_{m}=g_{m}$.

6. Prime ideals. An ideal $P$ is prime if $g h \in P$ implies $g \in P$ or $h \in P$. A $z$-filter is prime if, whenever the union of two zero-sets is a member, one of them is; here, it is sufficient to consider such unions equal to $X$ itself. The $z$-filter of any prime ideal is prime; and every prime $z$-filter is the $z$-filter of some prime ideal [2, Chapter 2].

An ideal $I$ is called a $\boldsymbol{z}$-ideal if $\boldsymbol{Z}(f) \in \boldsymbol{Z}[I]$ implies $f \in I$.

6.1. Theorem. A nonmaximal, prime z-ideal cannot be countably (z-) generated. ${ }^{7}$

Proof. Let $Q$ be such an ideal; we assume that $Q=\boldsymbol{Z}[Q]$ is countably generated and derive a contradiction. By Corollary $4.5, Q$ is a fixed ideal; let $M_{p}$ be the unique maximal ideal containing $Q$. Then $\{p\}=\phi(Q)$ is a countable intersection of zero-sets (2), and hence is a zero-set. Define

$$
S=X-\{p\}
$$

and let $P$ denote the trace of $\mathcal{Q}$ on $S$. Since every member of $Q$ contains $p$, the trace mapping

$$
Z \rightarrow Z-\{p\}
$$

from $Q$ onto $P$ is one-one.

Since the $z$-ideal $Q$ is not maximal, the zero-set $\{p\}$ does not belong to $Q$; therefore $\varnothing \notin \odot$. If $F$ is any zero-set in $S$, then $F \cup\{p\}$ is a zero-set in $X$ (see $[2,3 \mathrm{C}]$, or, more generally, $[3,3.1]$ ). These facts imply easily that $\rho$ is a $z$-filter on $S$, and, moreover, that it is prime. ${ }^{8}$ Obviously, $P$ is free. Since the trace mapping is one-one and preserves inclusion and intersection, $\odot$, like $Q$, is countably generated. But this contradicts Corollary 4.5.

In the family of all prime ideals in $C$, a prime ideal $Q$ with an immediate successor [predecessor] (under set inclusion) is called a lower [upper] ideal. Either neighbor of $Q$, when it exists, is unique; the

${ }^{7}$ This theorem is not vacuous. For hordes of fixed, nonmaximal prime $z$-ideals, see $[2,14 \mathrm{GHI}]$.

${ }^{8}$ For additional information, see $[5,2.2]$. 
first because the successors of $Q$ form a chain $[2,14.3(\mathrm{c})]$, the second by virtue of $[2,14.11]$. Unions and intersections of chains of prime ideals are, of course, prime. If $P \subset M^{p}$ is prime and $f \in M^{p}-P$, then $P^{f}$, the smallest prime ideal containing $(P, f)$, is an upper ideal; its associated lower ideal, $P_{f}$, is the largest prime ideal containing $P$ but not $f$.

6.2. Example. A fixed, countably generated, nonmaximal prime ideal. Let $\Sigma$ be the subspace $\mathbf{N} \cup\{\sigma\}$ of $\beta \mathbf{N}$, where $\sigma \in \beta \mathbf{N}-\mathbf{N}$, and let $f(n)=1 / n(n \in \mathrm{N}), f(\sigma)=0$. The ideal $O^{\sigma}$ in $C(\Sigma)$ is prime $[2,6.10]$, $f \in M^{\sigma}-O^{\sigma}$, and $O^{\sigma} \subset(f)[2,1 \mathrm{D}]$. By $[2,14 \mathrm{D}],\left(f, f^{1 / 2}, \cdots\right)$ is the prime ideal $\left(\boldsymbol{O}^{\sigma}\right)^{f}$.

6.3. Theorem. Every nonmaximal, countably generated, prime ideal is the union of a countable chain of upper ideals, and hence cannot be a countable intersection of lower ideals.

Proof. Let $Q=\left(f_{1}, f_{2}, \cdots\right)$ be such an ideal. Since $Q$ is not a $z$-ideal (6.1), it contains a prime ideal $P \neq Q[2,14.7]$. Then $Q=\cup_{n} P^{f_{n}}$, the union of a countable chain of upper ideals. The remaining implication is $[2,14.18]$.

6.4. Corollary. The intersection of a strictly decreasing sequence of prime ideals cannot be countably generated.

Proof. Consecutive members of the sequence have a lower ideal between them.

6.5. Example. A countably z-generated, lower prime ideal. $[2,2 \mathrm{G}]$ describes a nonmaximal prime ideal $P$ in $C(\mathbf{R})$ whose $z$-filter contains $\{0\}$. By $[2,14 \mathrm{D}], P$ is a lower ideal.

6.6. REMARK. ${ }^{9}$ Any finitely generated prime ideal in $C$ is trivial: it is minimal $[2,14 \mathrm{C}]$, hence a $z$-ideal $[2,14.7]$, hence maximal $(6.1)$, hence trivial (5.4).

\section{REFERENCES}

1. L. Gillman and M. Henriksen, Concerning rings of continuous functions, Trans. Amer. Math. Soc. vol. 77 (1954) pp. 340-362.

2. L. Gillman and M. Jerison, Rings of continuous functions, Princeton, Van Nostrand, 1960.

3. - Quotient fields of residue class rings of function rings, to appear in Illinois J. Math.

4. C. W. Kohls, Prime ideals in rings of continuous functions, Illinois J. Math. vol. 2 (1958) pp. 505-536.

5. - Prime ideals in rings of continuous functions, II, Duke Math. J. vol. 25 (1958) pp. 447-458.

InSTITUTE FOR ADVANCEd STUdy

${ }^{9}$ This observation is due to the referee. 